\title{
On the glycaemic index again
}

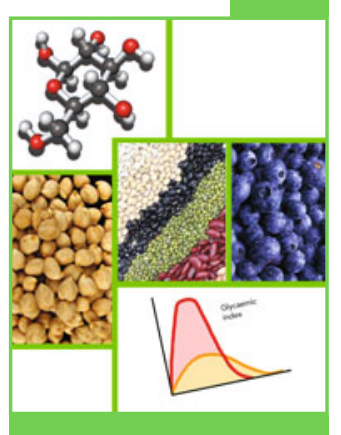

Correspondence to:

(C) Springer Healthcare - CEC Editore 2013

Different carbohydrates have very different effects on post-prandial glycaemia. In the past few years, researchers have paid considerable attention to this phenomenon, usually referred to as "glycaemic response". Even the European Food Safety Authority (EFSA), which is notoriously cautious in its evaluation of physiological food-related effects, recently accepted the notion that a glycaemic response reduction is salubrious and can, therefore, be exhibited as a "health claim". This view is very similar to that outlined in the Consensus document on "glycaemic index and glycemic load" recently drafted at the International Scientific Consensus Summit in Stresa, Italy (June 2013) and discussed in a recent editorial in Nutrafoods [1].

This issue of Nutrafoods features two very interesting papers on the health effects of carbohydrates, in relation to their glycaemic indexes. The first one, by Livia Augustin (of the research group directed by David Jenkins who developed the concept of the glycaemic index in the early 1980s), reviews the ex- perimental and clinical data that underscore the importance of maintaining a low glycaemic index/load for optimal health and for the prevention of specific disorders (especially, but not exclusively, type 2 diabetes, coronary heart disease and some types of cancer). The second one, by Anke Sentko (chief scientist of an important German company), discusses two potential approaches to controlling glycaemic response already available on the market. This shows how the most forward-thinking companies can contribute to our health via the development of appropriate food items or ingredients.

It is worth noting that an expert committee convened by David Jenkins and Walter Willett after the Stresa Consensus Meeting, the "International Carbohydrate Quality Consortium" (ICQC), also finds this interaction between basic research and food companies very interesting. Consequently, specific projects are being implemented to better understand the health effects of specific carbohydraterich foods. 